\title{
Kosterlitz-Thouless Universality in Dimer Models
}

\author{
Shailesh Chandrasekharan and Costas G. Strouthos \\ Department of Physics, Box 90305, Duke University, Durham, North Carolina 27708, USA.
}

\begin{abstract}
Using the monomer-dimer representation of strongly coupled $U(N)$ lattice gauge theories with staggered fermions, we study finite temperature chiral phase transitions in $(2+1)$ dimensions. A new cluster algorithm allows us to compute monomer-monomer and dimer-dimer correlations at zero monomer density (chiral limit) accurately on large lattices. This makes it possible to show convincingly, for the first time, that these models undergo a finite temperature phase transition which belongs to the Kosterlitz-Thouless universality class. We find that this universality class is unaffected even in the large $N$ limit. This shows that the mean field analysis often used in this limit breaks down in the critical region.
\end{abstract}

Computing quantities in Lattice QCD with massless quarks is notoriously difficult. Most known algorithms break down in the chiral limit. For this reason questions related to the universality of chiral phase transitions are among the many questions that remain unanswered. It is often difficult to compute critical exponents sufficiently accurately to rule out all possibilities except one.

The most useful simplification of lattice QCD occurs in the strong coupling limit which retains much of the underlying physics of QCD except for large lattice artifacts. In this limit spontaneous chiral symmetry breaking and its restoration due to finite temperature effects have been studied using large $N$ and large $d$ expansions [1-3]. However, since these approaches are based on mean field analysis they cannot help in determining the universality of phase transitions.

Interestingly lattice QCD with staggered fermions interacting through $U(N)$ gauge fields can be mapped into a monomer-dimer system in the strong coupling limit [4]. These models contain an exact $U(1)$ chiral symmetry, a remnant of the full chiral symmetry of QCD. When it was proposed, the monomer-dimer representation offered a new approach to study strongly coupled gauge theories close to the chiral limit from first principles. Unfortunately, this dream has remained unfulfilled until now. As in the weak coupling regime, most numerical simulations of the monomer-dimer systems have suffered from critical slowing down close to the chiral limit and hence have only allowed calculations with limited accuracy [5].

Recently, a cluster algorithm has been discovered to study these strongly coupled lattice gauge theories in the chiral limit [6]. This allows precision calculations in the chiral limit for the first time. As a first application of this new algorithm, in this article we study the finite temperature critical behavior in $(2+1)$ dimensions. In agreement with expectations from universality, we find with very high precision the chiral phase transition to be in the same universality class as the Berezinski-KosterlitzThouless (BKT) transition [7]. Our results are comparable to other known high precision spin-model studies of this universality class. We also show that the BKT transition persists even in the large $N$ limit, showing that the mean field analysis breaks down in the critical region.

Although our results will primarily be of interest to lattice gauge theorists, it is possible that they may have a wider audience. We are basically studying monomerdimer models and such models have a long history $[8,9]$. They have been studied extensively in the context of statistical and condensed matter physics. In the sixties, these models attracted a lot of attention $[10,11]$ when it was shown that the Ising model can be rewritten as a dimer model [12]. In the late eighties, these models became fashionable again in the quantum version [13] as a promising approach to the famous Resonating-ValenceBond (RVB) liquid phase [14]. More recently the quantum dimer approach has again gained momentum since it was shown that the RVB liquid phase is actually realized on a triangular lattice [15]. This has raised hope that dimer models may also yield a theoretical understanding of the spin-liquid phase [16]. Inspite of these studies, as far as we know, a BKT transition has not been studied in the context of a dimer model.

The partition function of the model we study in this article is given by ([4])

$$
Z=\int[d U] d \psi d \bar{\psi} \exp (-S[U, \psi, \bar{\psi}])
$$

where $[d U]$ is the Haar measure over $U(N)$ matrices and $d \psi d \bar{\psi}$ specify Grassmann integration. At strong couplings, the Euclidean space action $S[U, \psi, \bar{\psi}]$ is given by the fermionic part

$$
-\sum_{\langle i j\rangle} \eta_{\langle i j\rangle}\left[\bar{\psi}_{i} U_{\langle i j\rangle} \psi_{j}-\bar{\psi}_{j} U_{\langle i j\rangle}^{\dagger} \psi_{i}\right]-m \sum_{i} \bar{\psi}_{i} \psi_{i}
$$

where $i$ refers to the lattice site on a periodic three dimensional cubic lattice of size $L_{x}, L_{y}$ along the two spatial directions and size $L_{t}$ along the euclidean time direction, $\langle i j\rangle$ represents the bond connecting the nearest neighbor sites $i$ and $j, U_{\langle i j\rangle}$ are $N \times N$ unitary matrices associated with the bond $\langle i j\rangle$ and represent the gauge fields, $\psi_{i}$ is an $N$ component column vector and $\bar{\psi}_{i}$ is an $N$ component row vector made up of Grassmann variables and represent staggered fermion fields at the site $i$. We will assume that the gauge links satisfy periodic boundary 
conditions while the fermion fields satisfy either periodic or anti-periodic boundary conditions. The factors $\eta_{\langle i j\rangle}$ are the well known staggered fermion phase factors that depend on the coordinates $i$ (or $j$ ). We will choose them to have the property that $\eta_{\langle i j\rangle}^{2}=1$ when $\langle i j\rangle$ is a spatial bond, and $\eta_{\langle i j\rangle}^{2}=T$ when $\langle i j\rangle$ is a temporal bond. The exact form of $\eta_{\langle i j\rangle}$ is not important at strong couplings since only $\eta_{\langle i j\rangle}^{2}$ appears in the final partition function. The real parameter $T$ acts like a temperature. By working on asymmetric lattices with $L_{t}<<L$ and allowing $T$ to vary continuously, one can study finite temperature phase transitions in strong coupling QCD [5].

The partition function given in eq.(1) can be rewritten as a monomer-dimer system given by

$$
Z=\sum_{[n, b]}^{\prime} \prod_{\langle i j\rangle}\left(z_{\langle i j\rangle}\right)^{b_{\langle i j\rangle}} \frac{\left(N-b_{\langle i j\rangle}\right) !}{b_{\langle i j\rangle} ! N !} \prod_{i} \frac{N !}{n_{i} !} m^{n_{i}}
$$

and is discussed in detail in $[4,6]$. Here $n_{i}=0,1,2, \ldots, N$ refers to the number of monomers on the site $i, b_{\langle i j\rangle}=$ $0,1,2, \ldots, N$ represents the number of dimers on the bond $\langle i j\rangle, m$ is the monomer weight, $z_{\langle i j\rangle}=\eta_{i j}^{2}$ are the dimer weights. Note that while spatial dimers carry a weight 1 , temporal dimers carry a weight $T$. The sum is over all monomer-dimer configurations $[n, b]$ which are constrained such that the sum of the number of monomers at each site and the dimers that touch the site is always $N$ (the number of colors). The ' in the sum reminds us of this constraint. In this work we choose $L_{x}=L_{y}=L$.

Comparing eqs. (3) and (1) we learn that zero monomer density corresponds to the chiral limit. The bipartite nature of the lattice can be used to distinguish every site $j$ as either even or odd. If we define $\sigma_{j}=+1$ for an even site and $\sigma_{j}=-1$ for an odd site, it is easy to show that when $m=0$ the action given in eq. (2) is invariant under $U(1)$ chiral transformations,

$$
\psi(j) \rightarrow \mathrm{e}^{i \sigma_{j} \theta} \psi(j), \quad \bar{\psi}(j) \rightarrow \bar{\psi}(j) \mathrm{e}^{i \sigma_{j} \theta} .
$$

The results of [6] show convincingly that this $U(1)$ chiral symmetry breaks spontaneously at zero temperatures in two spatial dimensions. In this article we study the finite temperature critical behavior and show that it belongs to the BKT universality class as expected. For this purpose we compute the chiral susceptibility $\chi_{s}$ and the winding number susceptibility $\chi_{W}$, both of which are often computed in this context. The chiral susceptibility is equivalent to the spin susceptibility of classical $X Y$ models and is given by

$$
\chi_{s}=\frac{1}{V} \sum_{i, j}\left\langle\bar{\psi}_{i} \psi_{i} \bar{\psi}_{j} \psi_{j}\right\rangle=\left.\frac{1}{V Z} \frac{\partial^{2} Z}{(\partial m)^{2}}\right|_{m=0}
$$

where $V=L^{2} L_{t}$ is the lattice volume. It is easy to see that $\chi_{s}$ is the integrated monomer-monomer correlation function. We define the winding number susceptibility by

$$
\chi_{W}=\frac{\pi}{2}\left\langle W_{x}^{2}+W_{y}^{2}\right\rangle .
$$

where $W_{x}$ and $W_{y}$ are the winding numbers in the $x$ and $y$ directions respectively and are given by $W_{x}=$ $\left(\sum_{i} \sigma_{i} b_{\langle i j\rangle}\right) / L_{x}$, and $W_{y}=\left(\sum_{i} \sigma_{i} b_{\langle i j\rangle}\right) / L_{y}$. In the definition of $W_{x}$ and $W_{y}$ the site $j$ is chosen such that the bond $\langle i j\rangle$ is along the positive $x$ and $y$ directions respectively.

In this study we fix $L_{t}$ and compute $\chi_{s}$ and $\chi_{W}$ as a function of $L$. There are striking predictions for the large $L$ behavior of these quantities if the phase transition belongs to the BKT universality class. If $T_{c}$ represents the critical temperature we expect

$$
\chi_{s} \propto \begin{cases}L^{2-\eta(T)} & T<T_{c} \\ L^{1.75}[\log (L)]^{0.125} & T=T_{c} \\ \text { constant } & T>T_{c}\end{cases}
$$

and

$$
\chi_{W}= \begin{cases}1 /[2 \eta(T)] & T<T_{c} \\ {\left[2+1 / \log \left(L / L_{0}\right)\right]} & T=T_{c} \\ 0 & T>T_{c}\end{cases}
$$

in the large $L$ limit. At $T=T_{c}$ the next to leading order correction is also shown since it can be important. The critical exponent $\eta(T)$ is expected to change continuously with temperature but remain in the range $0 \leq \eta(T)<$ 0.25 assuming the monomers carry a unit of $U(1)$ charge. These predictions have been discussed in [7, 17-19] and have been used in $[20,21]$ earlier to demonstrate BKT behavior. In order to confirm these predictions we have computed $\chi_{s}$ and $\chi_{W}$ using the algorithm discussed in [6], for lattice sizes ranging from $L=32$ to $L=750$ and the number of colors from $N=1$ to $N=32$. We vary $T$ with fixed $L_{t}=4$ to study the critical behavior.

Let us first discuss our results for $N=1$. In figures 1 and 2 we plot $\chi_{s}$ and $\chi_{W}$ as functions of $L$. We find that $\chi_{s}$ fits extremely well to the form $b L^{2-\eta}$ when $T \leq 1.0$ with $\eta$ changing continuously in the expected region. In table I we show the fits along with their $\chi^{2}$ per degree of freedom $\left(\chi^{2} /\right.$ d.o.f. $)$. In the last column we give the results for $1 /\left(2 \chi_{W}\right)$ on the largest lattices that we could compute it. This value matches the value of $\eta$ obtained by the fits. The figures and the fits also indicate that when $T \geq 1.10$ one is in the high temperature phase with a finite correlation length.

When $1.02 \leq T \leq 1.06$, we find that the values of $\eta$ do not match $1 / 2 \chi_{W}$ even on the largest lattices while their values are close to a quarter as expected near the phase transition. There are noticeable finite size effects in $\chi_{W}$. Assuming that these discrepancies are due to the logarithmic corrections we fit the data for $\chi_{s}$ to the form $b L^{2-\eta}(\log (L))^{-2 r}$. In table II we show these new fits. We 


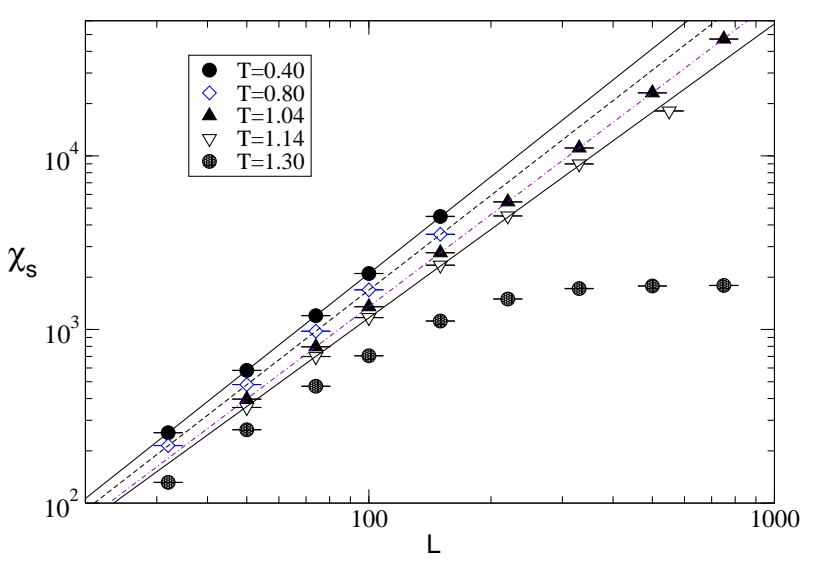

FIG. 1: Plot of $\chi_{s}$ vs. $L$ for various values of $T$.

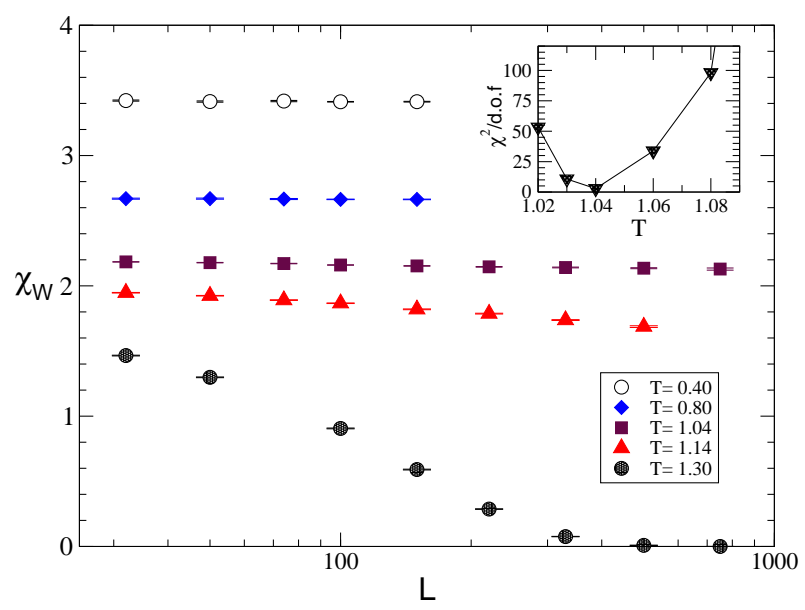

FIG. 2: The plot of $\chi_{W}$ as a function of $L$ for various values of $T$. The inset shows $\chi^{2} /$ d.o.f vs. $T$ for the fit $\chi_{W}=2+$ $1 / \log \left(L / L_{0}\right)$ which is valid only at $T_{c}$.

also fit the data for $\chi_{W}$ to the form $\left(c+1 / \log \left(L / L_{0}\right)\right)$ following [20]. The last column of table II shows the values of $1 / 2 c$ obtained from the fit. We find that the logarithmic terms are unimportant for $T \leq 1.0$ as expected. However, they appear to affect the value of $\eta$ and $1 /(2 c)$ (which is $1 /\left(2 \chi_{W}\right)$ in the thermodynamic limit) in the range $1.02 \leq T \leq 1.06$. In particular the value of $2 r$ is consistent with expectations at $T=1.04$. Further, the new values of $\eta$ are closer to $1 / 2 c$. Using the fact that $\chi_{W}=\left(2+1 / \log \left(L / L_{0}\right)\right)$ is exactly valid at $T=T_{c}$ we fit the data for $\chi_{W}$ to this form for various values of $T$. In the inset of figure 2 we plot $\chi^{2} /$ d.o.f as a function of $T$. Based on where the minimum occurs we estimate $T_{c}=1.040(5)$.

We have checked that $\chi_{s}$ and $\chi_{W}$ show similar evi-

\begin{tabular}{|c|c|c|c|c|}
\hline$T$ & $b$ & $\eta$ & $\chi^{2} /$ d.o.f & $1 /\left(2 \chi_{W}\right)$ \\
\hline 0.40 & $0.413(2)$ & $0.147(1)$ & 0.5 & $0.147(1)$ \\
0.80 & $0.401(2)$ & $0.188(1)$ & 2.4 & $0.189(1)$ \\
1.00 & $0.396(1)$ & $0.2236(5)$ & 0.2 & $0.2256(7)$ \\
1.02 & $0.397(1)$ & $0.2293(4)$ & 1.5 & $0.2321(7)$ \\
1.04 & $0.400(1)$ & $0.2356(4)$ & 2.0 & $0.2378(8)$ \\
1.06 & $0.402(1)$ & $0.2421(5)$ & 0.7 & $0.246(1)$ \\
1.10 & $0.413(1)$ & $0.2604(4)$ & 6.5 & $0.269(1)$ \\
1.14 & $0.463(1)$ & $0.3005(6)$ & 693 & 0.0000 \\
\hline
\end{tabular}

TABLE I: Fits for $\chi_{s}=b L^{2-\eta}$. The last column gives the value of $1 / 2 \chi_{W}$ on the largest lattices.

\begin{tabular}{|c|c|c|c|c|c|}
\hline$T$ & $b$ & $\eta$ & $2 \mathrm{r}$ & $\chi^{2} /$ d.o.f & $1 /(2 c)$ \\
\hline 1.00 & $0.398(5)$ & $0.222(5)$ & 0 & 0.2 & $0.2343(8)$ \\
1.02 & $0.391(5)$ & $0.235(5)$ & $-0.3(2)$ & 1.5 & $0.2411(5)$ \\
1.04 & $0.384(5)$ & $0.251(5)$ & $-0.07(2)$ & 0.4 & $0.2483(5)$ \\
1.06 & $0.395(5)$ & $0.249(5)$ & $-0.03(2)$ & 0.5 & $0.2583(5)$ \\
1.10 & $0.285(4)$ & $0.388(5)$ & $-0.12(2)$ & 3.6 & $0.2831(5)$ \\
1.14 & $0.243(4)$ & $0.569(6)$ & $-1.24(3)$ & 480 & - \\
\hline
\end{tabular}

TABLE II: Fits for $\chi_{s}=b L^{2-\eta}(\log (L))^{-2 r}$. The last column gives the value of $1 / 2 c$ obtained from the fits to $\chi_{W}$ discussed in the text.

dence for a BKT transition at higher values of $N$. Using techniques similar to the $N=1$ we have computed $T_{c}$ for various values of $N$. We find that $T_{c}=$ $0.708(6) N+1.40(4)-1.07(4) / N$ fits our results very well for all values of $N$ with a $\chi^{2} /$ d.o.f of 1.1. The detailed analysis of results at higher $N$ and the dependence of non-universal critical properties on $L_{t}$ will be presented elsewhere.

With regards to the $N$ dependence of our results we find two interesting observations. When $N$ approaches infinity it is often believed that a mean field analysis becomes useful. Here we argue that there are exceptions to this lore. Consider the Mermin-Wagner-Coleman theorem which shows that a continuous symmetry cannot break in two dimensions [22]. However, it can be shown that $\eta=0$ at $N \rightarrow \infty$ in the mean field approach, which means that the condensate is non-zero. Clearly, this cannot be true at any large but finite $N$. Witten has argued that when the symmetry is $U(1)$ the large $N$ analysis is still applicable since $\eta \sim 1 / N$ at large $N$ [23]. Our results for a fixed $T=1.0$, shown in figure 3 , do agree with this conjecture. Interestingly, as $N$ becomes large and $T / T_{c}$ is held fixed instead of $T$, we find that $\eta \neq 0$ even in the large $N$ limit. Figure 4 shows that $\eta$ approaches an interesting function of $T / T_{c}$ as $N$ becomes large. Extending this observation to QCD, we think that the t'Hooft limit (large N, small gauge coupling $g$, with $g^{2} N$ held fixed) may be quite similar [24].

In strictly two dimensions the monomer-monomer cor- 


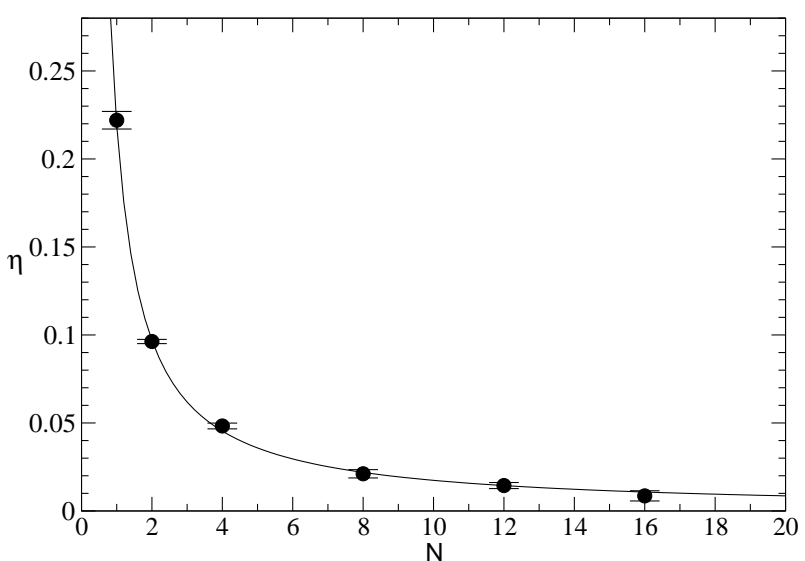

FIG. 3: The plot of $\eta$ as a function of $N$ for $T=1$. The data fits very well to the form $\eta=0.169(6) / N+0.050(9) / N^{2}$ with $\chi^{2} /$ d.o.f. $=1.2$.

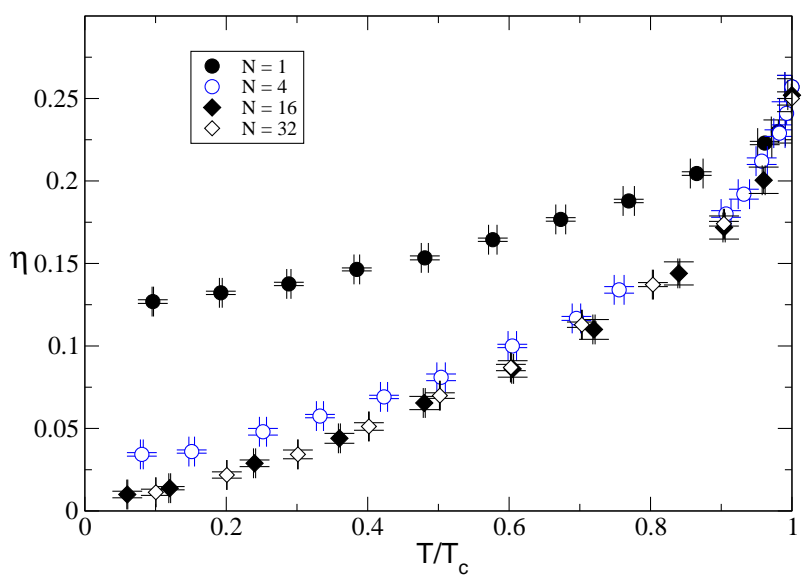

FIG. 4: Plot of $\eta$ as a function of $T / T_{c}$.

relations for $N=1$ were calculated in $[11,25]$. It was found that $\eta \sim 0.5$, which was recently confirmed again in [6]. This is not in the range $0 \leq \eta<0.25$ we found above. We think a closer examination of this result in the context of BKT transitions would be interesting.

Finally we note that the $N=1$ model has been recently studied in [26] on various types of lattices, using another algorithm which is efficient in measuring dimerdimer correlations at zero monomer density [27]. The authors of [26] show the existence of a Coulomb phase in three dimensions on cubic lattices. Our results support this observation indirectly, however we interpret the results differently. We use the connection of the dimer model with strongly coupled lattice QED to show that the long range correlations are a result of a spontaneous breaking of a global $U(1)$ chiral symmetry in three dimensions. The winding numbers $W_{x}$ and $W_{y}$ we have defined are exactly the magnetic flux in the $x$ and the $y$ directions obtained using the magnetic field defined in [26]. We interpret the divergence free magnetic field as the conserved current related to the $U(1)$ chiral symmetry.

We would like to thank P. Hasenfratz, R. Moessner, S. Sondhi and U.-J. Wiese for useful comments. This work was supported in part by the National Science Foundation grant DMR-0103003 and the U.S. Department of Energy grant DE-FG-96ER40945.

[1] N. Kawamoto and J. Smit, Nucl. Phys. B192, 100 (1981).

[2] H. Kluberg-Stern, A. Morel and B. Petersson, Nucl. Phys. B215, 527 (1983).

[3] P.H. Damgaard, N. Kawamoto and K. Shigemoto, Phys. Rev. Lett. 53, 2211 (1984).

[4] P. Rossi and U. Wolff, Nucl. Phys. B248, 105 (1984).

[5] G. Boyd et. al., Nucl. Phys. B376, 199 (1992).

[6] S. Chandrasekharan and D.H. Adams, Nucl. Phys. B662, 220 (2003).

[7] V.L. Berezinski, Sov. Phys. JETP 34, 610 (1971); J.M. Kosterlitz and D.J. Thouless, J. Phys. C6, 1181 (1973).

[8] R. S. Fowler and G.S. Rushbrook, Trans. Faraday Soc., 331272 (1937).

[9] O. J. Heilmann and E. H. Lieb, Comm. Math. Phys. 25, 190 (1972).

[10] P. W. Kasteleyn, Physica 27, 1209 (1961); J. Math. Phys. 4, 287 (1963).

[11] M. Fisher and J. Stephenson, Phys. Rev. 132, 1411 (1963);

[12] M. E. Fisher, J. Phys. 7, 1776 (1966).

[13] D. S. Rokhsar and S. A. Kivelson, Phys. Rev. Lett. 61, 2376 (1998).

[14] P. W. Anderson, Science 235, 1196 (1987).

[15] R. Moessner and S.L. Sondhi, Phys. Rev. Lett 86, 1881 (2001).

[16] R. Moessner, S.L. Sondhi and E. Fradkin, Phys. Rev. B65, 024504 (2002).

[17] R. Kenna and A.C. Irving, Phys. Lett. B351, 273 (1995).

[18] D.R. Nelson and J. M. Kosterlitz, Phys. Rev. Lett. 39, 1201 (1977).

[19] E.L. Pollock and D.M. Ceperley, Phys. Rev. B36, 8343 (1987).

[20] K. Harada and N. Kawashima, J. Phys. Soc. Jpn. 67, 2768 (1998).

[21] S. Chandrasekharan and J. C. Osborn, Phys. Rev. B 66, 045113 (2002).

[22] N. D. Mermin and H. Wagner, Phys. Rev. Lett. 17, 1133 (1966); S. Coleman, Comm. Math. Phys. 31, 259 (1973).

[23] E. Witten, Nucl. Phys. B145, 110 (1978).

[24] t'Hooft, Nucl. Phys. B72, 461 (1974).

[25] R. E. Hartwig, J. Math. Phys. 7, 286 (1966).

[26] D. A. Huse, W. Krauth, R. Moessner, S. L. Sondhi, arXiv:cond-mat/0305318.

[27] W. Krauth and R. Moessner, arXiv:cond-mat/0206177. 